\title{
Analysis of erythrocyte glycophorin-A variants by flow cytometry in lung disease patients detects the effect of tobacco smoke
}

\author{
Monica Neri ${ }^{\mathrm{a}, *}$, Elio Geido ${ }^{\mathrm{b}}$, \\ Rosangela Filiberti ${ }^{a}$, Roberto Orecchia ${ }^{\mathrm{b}}$, \\ Angela Di Vinci ${ }^{b}$, Mara Cafferata ${ }^{c}$, \\ Elisabetta Tassara ${ }^{\mathrm{d}}$, Riccardo Puntoni ${ }^{\text {a }}$ \\ and Walter Giaretti ${ }^{\mathrm{b}}$

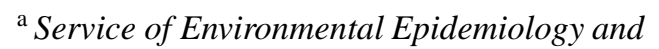 \\ Biostatistics, National Cancer Research Institute, \\ Genoa, Italy \\ ${ }^{\mathrm{b}}$ Laboratory of Biophysics-Cytometry, Department of \\ Preventive Oncology, National Cancer Research \\ Institute, Genoa, Italy \\ c Service of Medical Oncology, National Cancer \\ Research Institute, Genoa, Italy \\ ${ }^{\mathrm{d}}$ Service of Thoracic Endoscopy, National Cancer \\ Research Institute, Genoa, Italy
}

Received 21 September 2000

Accepted 10 November 2000

The glycophoryn A (GPA) assay evaluates somatic in vivo mutations. It is considered a cumulative biodosimeter for genotoxic exposures and is under evaluation in cancer risk assessment.

GPA, a polymorphic membrane protein of the erythrocytes, determines the MN blood groups. The NO and NN variant frequencies (VF) may be detected in MN subjects (about $50 \%$ of the population) by flow cytometry using two differently labelled antibodies.

We explored if GPA N0 and NN VF might be relevant to the assessment of individual lung cancer risk and susceptibility, in a small population with a high prevalence of heavy tobacco smokers: 8 lung cancer patients and 16 subjects with non-malignant lung diseases associated with increased risk of lung cancer.

\footnotetext{
*Corresponding author: Dr. Monica Neri, PhD, Environmental Epidemiology and Biostatistics, National Cancer Research Institute, Largo Rosanna Benzi 10, 16132 Genoa, Italy. Tel.: +39 010 5600777; Fax: +39010 5600501; E-mail: nerim@ @p380.ist.unige.it.
}

There was a wide interindividual variability and complete overlap between non-neoplastic and neoplastic patients. A significant positive correlation was seen with smoking duration in N0 VF ( $p=0.04$, age-adjusted). Current smokers $(n=12)$ displayed higher N0 values than never $(n=1)$ or ex-smokers $(n=11), 36.3 \pm 18.2$ and $21.0 \pm 13.2$, respectively $(p<0.01)$. No association was shown with occupational exposure.

The present exploratory study suggests that assessment of individual lung cancer risk and susceptibility by the GPA assay does not seem to be feasible. The assay appears to provide a biomarker of longterm exposure to tobacco smoke.

\section{Introduction}

Mutations of a great number of genes are central in human cancer genesis and progression. These mutations involve not only oncogenes and tumor suppressor genes, which produce respectively dominant gain and recessive loss of function, but also other genes associated to DNA repair, apoptosis, and metastasis [13]. Knowledge of cancer associated somatic mutations in humans is important to understand the aetiology of cancer as well as to help in the assessment of cancer risk and to plan preventive interventions. This is particularly important for lung cancers, the great majority of which are caused by exogenous mutagenic exposures such as tobacco smoke and, to a lesser extent, occupational exposure [14].

Somatic in vivo mutation rates may be evaluated by use of reporter genes, that are not directly related to carcinogenesis, and using surrogate tissues, such as peripheral blood, that are more easily accessible than the target tissues.

Extensive databases are available for the assay that uses the glycophorin A (GPA) from red blood cells as the reporter gene [2,9]. The polymorphic glycoprotein GPA is present in the cell membrane of the erythrocytes at approximately $5 \times 10^{5}$ molecules per cell. The 
gene spans $44 \mathrm{~kb}$ on chromosome 4 and is a rather large target for mutations. The two allelic forms $\mathrm{M}$ and $\mathrm{N}$ determine the $\mathrm{MN}$ blood groups. The isoforms differ by two non-adjacent aminoacids, cannot be interconverted by simple point mutations and are codominantly expressed. About $50 \%$ of the human population are MN-heterozygous: their erythrocytes show both $\mathrm{M}$ and $\mathrm{N}$ molecules on the surface and are suitable for the GPA assay.

Simple loss mutations, mainly point mutations and deletions, may generate the hemizygous variants, N0 cells that have lost the expression of the $M$ form. Homozygous NN variants, instead, stem from loss of function of one allele with double expression of the other, due to chromosome missegregation and somatic recombination or gene conversion [11]. A flow cytometry (FCM) assay of GPA was proposed to detect the frequency of variant erythrocytes (VF) using fluorescent monoclonal antibodies specific for the $M$ and $\mathrm{N}$ molecules [17]. The mean variant frequency values for adults usually are in the order of magnitude of $10 \times 10^{-6}$, with a wide interindividual variability [2,9].

The GPA assay was proposed to provide an evaluation of the life-span cumulative exposure for humans, because it detects mutations preserved at the level of red blood stem cells during the lifetime of an individual. GPA variants were found to be at significantly increased frequency among Hiroshima A-bomb survivors; the assay was tentatively used as a biodosimeter of cumulative exposure after the fall-out in the Chernobyl nuclear power center $[2,5,9,16,18,26]$. The GPA assay by FCM was also used for monitoring the genotoxic load in cancer patients who underwent radio- or chemotherapy $[4,12,15,22,24,27]$, in workers exposed to styrene $[6,10]$ and, in association with DNA adducts, for foundry workers exposed to polycyclic aromatic hydrocarbons [25].

The significance of the GPA assay by FCM in cancer risk assessment is still under investigation [1]. So far, however, it has been shown that GPA variants are at increased frequency in some cancer prone genetic disorders [9], and that they may provide a marker of risk of secondary leukemia in patients treated for childhood acute lymphoblastic leukemia [8].

The present pilot study is part of a multicenter molecular epidemiologic research on pleuropulmonar cancer designed to identify biomarkers of cancer susceptibility and increased individual risk. The analysis was aimed at exploring by multiparameter FCM the frequencies of N0 and NN GPA variants in lung cancer patients and in subjects with non-malignant chronic lung diseases at increased risk of developing lung cancer. The role of tobacco smoke was evaluated, taking advantage of the heavy smoking habits of the subjects in our study.

\section{Materials and methods}

\subsection{Study population}

Blood samples were obtained by venipuncture from 8 primary lung cancer cases and 16 patients affected by bronco-pulmonar chronic obstruction (BPCO). The lung cancer patients were enrolled at diagnosis, prior to any treatment. All subjects gave their informed consent and were recruited at the National Cancer Institute and at the Division of Pneumology of S. Martino Hospital, Genoa. Control blood samples to set up the methodology were obtained from healthy volunteers.

Health history of subjects was ascertained from clinical records. Lung cancers were classified according to the TNM classification criteria of UICC [21]: 5 cases had stage IV disease and one case stage IIIb, while for two patients the stage was not specified. The histology was defined according to WHO [28]: there were two adenocarcinoma, two squamocellular carcinoma, two cases simply defined as "non small cell lung cancer", and two not specified.

The subjects were 17 males and one female. The mean age was 66.1 years for the cancer cases (range: 47-86) and 66.4 years for the non-neoplastic patients (40-87).

Personal interviews were performed by trained personnel using a standardized questionnaire. All subjects provided information on demographic data, health status, employments, family history of cancer, and past exposure to X-ray for clinical purposes. The questionnaire was particularly detailed on tobacco smoke exposure and included questions on: smoking status, age at smoking initiation, duration of smoking, number of cigarettes per day, exposure to environmental tobacco smoke. There were no cigar or pipe smokers in the study group.

\subsection{GPA assay}

Blood samples were serotyped using $\mathrm{M}$ and $\mathrm{N}$ antisera (Ortho Diagnostic Systems, Raritan, NY) to identify the individuals eligible for the GPA assay, which requires blood of the MN group. Formalin-fixed spherical erythrocyte were stored frozen in fetal calf serum 
containing $5 \%$ DMSO (Sigma) at $-80^{\circ} \mathrm{C}$ until analysis. GPA assay by FCM was conducted according to the method reported by Langlois et al. [19] with later modifications introduced by Jensen and Bigbee [17].

Immunolabelling of fixed cells was performed in staining buffer (SB) at room temperature. A $100 \mu \mathrm{l}$ aliquot of fixed cells was washed by centrifugation $(2 \mathrm{~min}$ at $1000 \mathrm{~g}$ ) and resuspended in $1 \mathrm{ml}$ of SB. The cells were incubated with $1 \mu \mathrm{g} / \mathrm{ml}$ BRIC157-FITC and $2 \mu \mathrm{g} / \mathrm{ml}$ 6A7-PE monoclonal antibodies (IBGRL Research Products, UK) for $1 \mathrm{~h}$ on a rotatory shaker in the dark. The directly labelled cells were washed twice and resuspended in $1 \mathrm{ml}$ of SB containing $10 \mu \mathrm{g} / \mathrm{ml}$ propidium iodide. Labelled samples can be stored at $4^{\circ} \mathrm{C}$ for three days before analysis.

FCM was performed with a FACS cell sorter (Becton Dickinson, Mountain View, CA) equipped with an argon-ion laser to acquire $1 \times 10^{6}$ cellular events gated on scattering signals at flow rate around 1500-2000 cells/s.

The GPA variant frequencies (N0 and $\mathrm{NN}$ ) were expressed as the number of variant erythrocytes per $10^{6}$ cells analysed, according to the method reported by Jensen and Bigbee [17].

\subsection{Statistics}

Means, medians and standard deviations were calculated for each group under study. Differences in the median values between the groups were checked using the Kruskall-Wallis test, while the Mann-Whitney U test was used for comparison of two groups. Spearman correlation method was used to correlate the VF with the variables of interest. Differences were considered statistically significant at $p<0.05$.

\section{Results}

Figure 1 shows two examples obtained with the BRIC 157-FITC and 6A7-PE antibodies GPA assay by FCM. First, the measurement of cell mixtures was done with equal numbers of unlabelled fixed heterozygous MN erythrocytes and either cells singly labelled with BRIC 157-FITC or cells singly labelled with 6A7$\mathrm{PE}$, in order to adjust the FACS photomultipliers gain and compensation settings (not shown). Next, a three cell control test mixture was used with fixed spherical erythrocytes from 3 blood donors (MM, NN and MN) mixed in equal amounts of $50 \mu \mathrm{l}$ each stained with both antibodies (Fig. 1(A)). The discrimination of

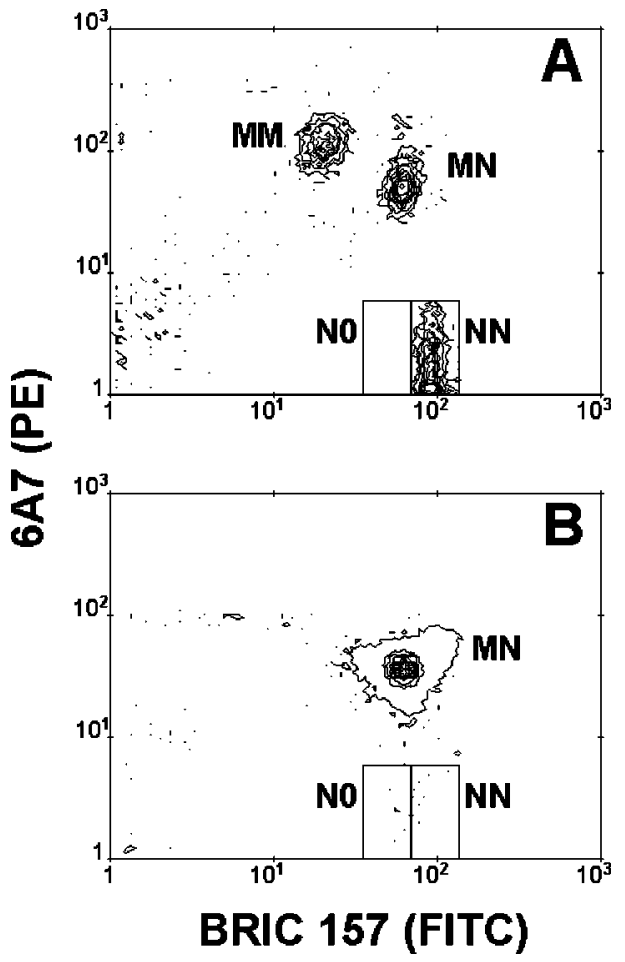

Fig. 1. Bivariate FCM distribution of a mixture at equal ratios of fixed erythrocytes obtained from $\mathrm{MM}, \mathrm{NN}$ and $\mathrm{MN}$ donors (A) and from a $\mathrm{MN}$ control healthy donor (B). The rectangular areas denoted by $\mathrm{N} 0$ and NN contain the corresponding GPA variant erythrocytes. The assay was performed by direct immunofluorescence labelling with specific monoclonal antibodies BRIC157 and 6A7.

N0 and NN variant erythrocytes from a MN individual is shown in Fig. 1(B) over 1 million cells. Variant N0 and NN erythrocytes were in this case $7 \times 10^{-6}$ and $8 \times 10^{-6}$, respectively.

The mean GPA variant frequencies among 16 individuals with bronco-pulmonar chronic obstruction and 8 with lung cancer were respectively $25.9 \pm 14.0$ and $33.9 \pm 23.4$ for N0 and $22.4 \pm 14.8$ and $17.9 \pm 11.1$ for $\mathrm{NN}\left(\mathrm{VF} \times 10^{-6}\right.$, means $\pm \mathrm{SD}$. See Table 1$)$. The mean values showed no statistically significant difference among the two groups of patients. The values were characterised by wide interindividual variability and coefficients of variation above $50 \%$.

The N0 and NN GPA variants were tested for association with all data obtained by questionnaire. No significant difference was found for age, occupational exposure (mainly asbestos or diesel exhaust), family history of cancer and clinical exposure to X-rays in the last 5 years prior to blood collection.

Smoking habits were analysed in detail (Table 2). Among the subjects, 4 declared to smoke less than 
Table 1

GPA NO and NN variant frequencies according to pathologies $\left(\mathrm{VF} \times 10^{-6}\right)$

\begin{tabular}{ccc}
\hline & N0 & NN \\
& $\begin{array}{c}\text { mean } \pm \text { SD } \\
\text { (range) }\end{array}$ & $\begin{array}{c}\text { mean } \pm \text { SD } \\
\text { (range) }\end{array}$ \\
\hline Lung cancer $(n=8)$ & $33.9 \pm 23.4$ & $17.9 \pm 11.1$ \\
& $(8-83)$ & $(7-43)$ \\
BPCO $^{*}(n=16)$ & $25.9 \pm 14.0$ & $22.4 \pm 14.8$ \\
& $(3-53)$ & $(7-50)$ \\
\hline
\end{tabular}

*Bronco-pulmonar chronic obstruction.

Table 2

GPA N0 and NN variant frequencies according to smoking habits $\left(\mathrm{VF} \times 10^{-6}\right)$

\begin{tabular}{lcc}
\hline & N0 & NN \\
& mean \pm SD & mean \pm SD \\
\hline Smoking status & & \\
$\quad$ Never or ex-smokers $(n=12)$ & $21.0 \pm 13.2$ & $23.9 \pm 13.3$ \\
Current smokers $(n=12)$ & $36.3 \pm 18.2$ & $18.7 \pm 13.6$ \\
& $p<0.01$ & \\
Duration (years) & & \\
$<41.5(n=12)$ & $23.7 \pm 15.0$ & $17.5 \pm 14.9$ \\
$\geqslant 41.5(n=12)$ & $33.7 \pm 18.9$ & $25.1 \pm 11.1$ \\
& $p=0.04$ & \\
Daily cigarette consumption & & \\
$<30(n=10)$ & $31.7 \pm 23.3$ & $17.3 \pm 12.1$ \\
$\geqslant 30(n=14)$ & $26.5 \pm 12.2$ & $24.1 \pm 14.0$ \\
\hline
\end{tabular}

20 cigarettes/day, 12 from 20 to 39 and 9 declared to smoke 40 or more cigarettes per day. The average intensity was 31.0 cigarettes per day (range 5-80). There was only one never smoker $(\mathrm{NO} \mathrm{VF}=20$; NN $\mathrm{VF}=27$ ).

Current smokers $(n=12)$ showed higher N0 values than never $(n=1)$ and ex-smokers (equal or greater than 1 year; $n=11): 36.3 \pm 18.2$ and $21.0 \pm 13.2$, respectively $(p<0.01)$. A positive association $(p=$ 0.04 ) was found with smoking duration (median 41.5 years) in GPA N0 variants, but not in NN, after adjusting for age and regardless the pathology.

No association was found with the intensity of cigarette smoking, neither when it was expressed as pack/years.

\section{Discussion}

Aim of this pilot study was to explore if the frequencies of N0 and NN GPA variants as detected by FCM might be relevant to the assessment of pleuropulmonar cancer susceptibility and increased individual risk in future investigations.

We compared individuals with lung cancer and with bronco-pulmonar chronic obstruction (BPCO). A number of non-malignant lung conditions, including BPCO, chronic bronchitis, emphysema, asthma, pneumonia, tubercolosis, silicosis and asbestosis have been associated with an increased risk of lung cancer [7]. Some of these conditions, such as BPCO, emphysema and chronic bronchitis, are strongly associated with tobacco smoking, but seem to increase the risk of lung cancer even in lifetime non-smokers [20,29].

Control data obtained from healthy donors were in general agreement with the frequencies usually reported for adults providing an order of magnitude of about $10 \times 10^{-6} \mathrm{~N} 0$ and NN GPA variants. A large interindividual variation was found with all the currently used reporter gene assays, including GPA [6]. It is considered to be mainly biological: haematopoietic stress, for example, may affect erythrocytes assay [2].

In the present study, we found that GPA variant frequencies were significantly associated with cigarette smoking habits, a condition that we have considered in detail in our questionnaire. Every other variable, including type of lung pathology and occupational exposure, was not associated. Current smokers had approximately $70 \%$ higher hemizygous VF than ex- or never smokers, and a positive trend existed with smoking duration. This seems to confirm and refine previous suggestions of an effect of smoking habit in the GPA assay $[3,9,16,27]$.

A small but significant effect of cigarette smoking on hemyzygous type VF was shown in a study on 1226 atomic bomb survivors, where the smoking effect was based on number of cigarettes smoked per day, with no information on duration [18].

Tobacco smoke was shown to be a potential confounder in a survey on workers exposed to styrene [10] and was subsequently analysed in detail in a molecular epidemiologic study on 47 Finnish reinforced plastics workers exposed to styrene and 47 matched controls [6]. Cigarette smoking was associated with significantly elevated GPA NO and NN variant frequencies among active smokers and among active and exsmokers combined, with respect to never smokers. Its influence on NO VF was particularly evident among active smokers in the control group. No information was reported on smoking duration, while no clear doseresponse effect was shown.

In our analysis an effect of tobacco smoke was apparent. The inclusion in our study of subjects affected 
by lung diseases, comprising a high proportion of longterm and heavy smokers, probably amplified it.

Frequencies of N0 and NN GPA variants were not statistically different between lung cancer cases and patients affected by BPCO. A great overlap of the individual values existed in the present series of subjects.

Increased frequencies of GPA variants were shown in patients with hepatocellular carcinoma with respect to healthy controls, while intermediate values were found in patients with chronic liver diseases predisposing to cancer [23]. In the literature, however, many studies reported overlapping values between non-neoplastic donors and patients affected by various cancers, including lung cancer $[1,4,8,12,24]$. Moreover, literature data, including our own, suggest that GPA hemizygous variants N0 carry a differential information with respect to homozygous $\mathrm{NN}$ variants. In fact, while NO cells may derive from simple point mutations and deletions, NN cells need also the duplication of one allele by mechanisms which are not yet well clear. Better knowledge of these mechanisms, including the process of chromosome missegregation and aneuploidy, as well as a more complete understanding of the carcinogenesis and progression of lung cancer is necessary.

The present study, though intended as exploratory and based only on a relatively small number of cases, provides data that, to our knowledge, are not yet present in the literature. Cancer predisposing diseases represent a rather unexplored field of investigation. Our present data suggest that the GPA assay by FCM may not help in the assessment of individual lung cancer risk in an at-risk population. The GPA assay, however, may offer a quick experimental approach to provide a potentially useful biomarker of early biological effect of the exposure to tobacco smoke.

\section{Acknowledgements}

This study was financially supported by the Italian Health Ministry and by the Italian Association for Cancer Research (A.I.R.C.).

\section{References}

[1] M. Akiyama, S. Umeki, Y. Kusunoki, S. Kyoizumi, N. Nakamura, T. Mori, Y. Ishikawa, M. Yamachido, K. Ohama, T. Kodama, K. Endo and J.B. Cologne, Somatic cell mutations as a possible predictor of cancer risk, Health Phys. 68 (1995), 643649.
[2] R.J. Albertini and R.B. Hayes, Somatic cell mutations in cancer epidemiology, IARC Scient. Publ. 142 (1997), 159-184.

[3] W.L. Bigbee, R.G. Langlois, M. Swift and R.H. Jensen, Evidence for an elevated frequency of in vivo somatic cell mutations in ataxia telangiectasia, Am. J. Hum. Genet. 44 (1989), 402-408.

[4] W.L. Bigbee, A.J. Wyrobeck, R.G. Langlois, R.H. Jensen and R.B. Everson, The effect of chemotherapy on the in vivo frequency of glycophorin A "null" variant erythrocytes, Mutat. Res. 240 (1990), 165-175.

[5] W.L. Bigbee, R.H. Jensen, T. Veidebaum, M. Tekkel, M. Rahu, A. Stengrevics, A. Auvinen, T. Hakulinen, K. Servomaa, T. Rytomaa, G.I. Obrams and J.D. Boice Jr, Biodosimetry of Chernobyl cleanup workers from Estonia and Latvia using the glycophorin A in vivo somatic cell mutation assay, Radiat. Res. 147 (1997), 215-224.

[6] W.L. Bigbee, S.G. Grant, R.G. Langlois, R.H. Jensen, A. Anttila, P. Pfaeffli, K. Pekari and H. Norppa, Glycophorin A somatic cell mutation frequencies in Finnish reinforced plastics workers exposed to styrene, Cancer Epidemiol. Biomarkers and Prev. 5 (1996), 801-810.

[7] W.J. Blot and J.F. Fraumeni Jr, Cancers of the lung and pleura, in: Cancer Epidemiology and Prevention, 2nd ed., D. Schottenfeld and J.F. Fraumeni Jr, eds, Oxford University Press, New York, NY, 1996

[8] J. Boyse, M. Hewitt and M.G. Mott, Glycophorin A mutations and risk of secondary leukaemia in patients treated for childhood acute lymphoblastic leukaemia, Brit. J. Haematol. 93 (1996), 117-124.

[9] J. Cole and T.R. Skopek, Somatic mutant frequency, mutation rates and mutational spectra in the human population in vivo, Mutat. Res. 304 (1994), 33-105.

[10] P.J. Compton-Quintana, R.H. Jensen, W.L. Bigbee, S.G. Grant, R.G. Langlois, M.T. Smith and S.M. Rappaport, Use of the glycophorin A human mutation assay to study workers exposed to styrene, Environ. Health Perspect. 99 (1993), 297-301.

[11] S.G. Grant and W.L. Bigbee, In vivo somatic mutation and segregation at the human glycophorin A (GPA) locus, Mutat. Res. 288 (1993), 63-172.

[12] S.G. Grant and W.L. Bigbee, Bone marrow somatic mutation after genotoxic cancer therapy, Lancet 343 (1994), 1507-1508.

[13] D. Hananan and R.A. Weinberg, The hallmarks of cancer, Cell 100 (2000), 57-70.

[14] IARC, Tobacco smoke, in: IARC Monographs on the Evaluation of the Carcinogenic Risks to Humans, Vol. 38, Lyon, 1986.

[15] R.H. Jensen, R.G. Langlois and W.L. Bigbee, Determination of somatic mutations in human erythrocytes by flow cytometry, Prog. Clin. Biol. Res. 209B (1986), 177-184.

[16] R.H. Jensen, W.L. Bigbee, R.G. Langlois, S.G. Grant, P.P. Pleshanov, A.A. Chirkov and M.A. Pilinskaya, Laser-based flow cytometric analysis of genotoxicity of humans exposed to ionizing radiation during the Chernobyl accident, in: Laser Applications in Life Sciences, SPIE Proceedings 1403 (1990), 372380.

[17] R.H. Jensen and W.L. Bigbee, Direct immunofluorescence labeling provides an improved method for the glycophorin A somatic cell mutation assay, Cytometry 23 (1996), 337-343. 
[18] S. Kyoizumi, M. Akiyama, J.B. Cologne, K. Tanabe, N. Nakamura, A.A. Awa, Y. Hirai, Y. Kusunoki and S. Umeki, Somatic cell mutations at the glycophorin A locus in erythrocytes of atomic bomb survivors: implications for radiation carcinogenesis, Radiat. Res. 146 (1996), 43-52.

[19] R.G. Langlois, B. Nisbet, W.L. Bigbee, D.N. Ridinger and R.H. Jensen, An improved flow cytometric assay for somatic mutations at the glycophorin A locus in humans, Cytometry 11 (1990), 513-521.

[20] S.T. Mayne, J. Buenconsejo and D.T. Janerich, Previous lung disease and risk of lung cancer among men and women nonsmokers, Am. J. Epidemiol. 149 (1999), 13-20.

[21] C.F. Mountain, A new international staging system for lung cancer, Chest (Suppl.) 89 (1986), 225-233.

[22] M.G. Mott, J. Boyse, M. Hewitt and M. Radford, Do mutations at the glycophorin A locus in patients treated for childhood Hodgkin's disease predict secondary leukaemia?, Lancet 343 (1994), 828-830.

[23] S. Okada, H. Ishii, H. Nose, T. Okusaka, A. Kyogoku, M. Yoshimori and K. Wakabayashi, Evidence for increased somatic cell mutations in patients with hepatocellular carcinoma, Carcinogenesis 18 (1997), 445-449.

[24] F.P. Perera, R.J. Motzer, D.L. Tang, E. Reed, R. Parker, D. Warburton, P. O'Neill, R. Albertini, W.L. Bigbee, R.H. Jensen, R. Santella, W.Y. Tsai, G. Simon-Cereijido, C. Randall and G. Bosl, Multiple biological markers in germ cell tumor patients treated with platinum-based chemotherapy, Cancer Res. 52 (1992), 3558-3565.
[25] F.P. Perera, D.L. Tang, J.P. O’Neill, W.L. Bigbee, R.J. Albertini, R. Santella, R. Ottman, W.Y. Tsai, C. Dickey, L.A. Mooney, K. Savela and K. Hemminki, HPRT and glycophorin A mutations in foundry workers: relationship to PAH exposure and to PAH-DNA adducts, Carcinogenesis 14 (1993), 969-973.

[26] A.S. Saenko, I.A. Zamulaeva, S.G. Smirnova, N.V. Orlova, E.I. Selivanova, V.A. Saenko, N.P. Mattveeva, M.A. Kaplan, V.Y. Nugis, N.M. Nadezhina and A.F. Tsyb, Determination of somatic mutant frequencies at glycophorin A and T-cell receptor loci for biodosimetry of prolonged irradiation, Int. J. Radiat. Biol. 73 (1998), 613-618.

[27] J. Schiwietz, L. Lorenz, M. Scheubeck, W. Boerner and K. Hempel, Improved determination of variant erythrocytes at the glycophorin A (GPA) locus and variant frequency in patients treated with radioiodine for thyroid cancer, Int. J. Radiat. Biol. 70 (1996), 131-143.

[28] WHO, Histological typing of lung tumors, Am. J. Clin. Pathol. 77 (1982), 123-136.

[29] A.H. Wu, E.T.H. Fontham, P. Reynolds, R.S. Greenberg, P. Buffler, N. Liff, P. Boyd, B.E. Henderson and P. Correa, Previous lung disease and risk of lung cancer among lifetime nonsmoking women in the United States, Am. J. Epidemiol. 141 (1995), 1023-1032. 


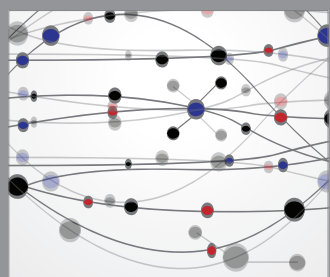

The Scientific World Journal
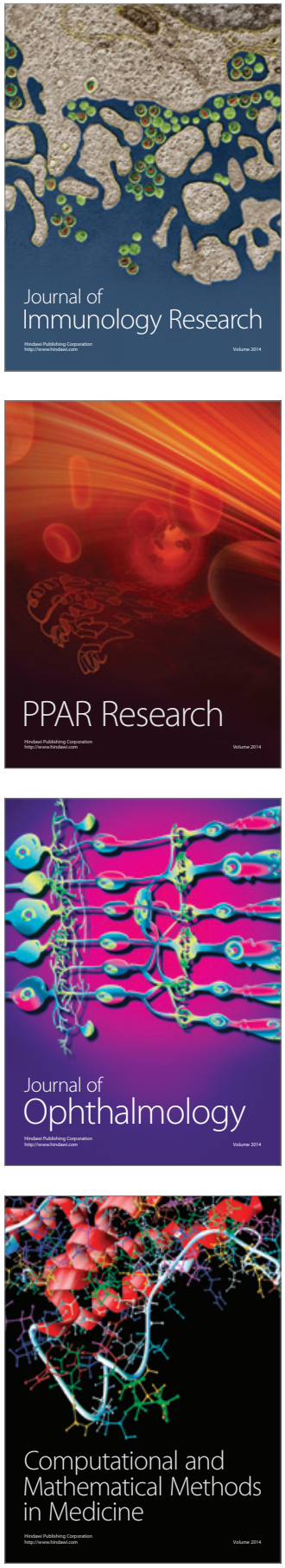

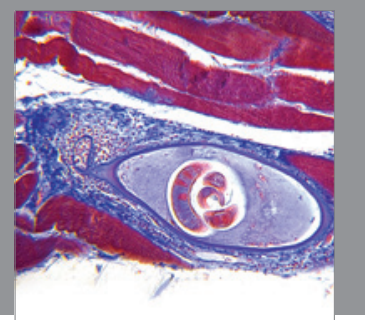

Gastroenterology

Research and Practice
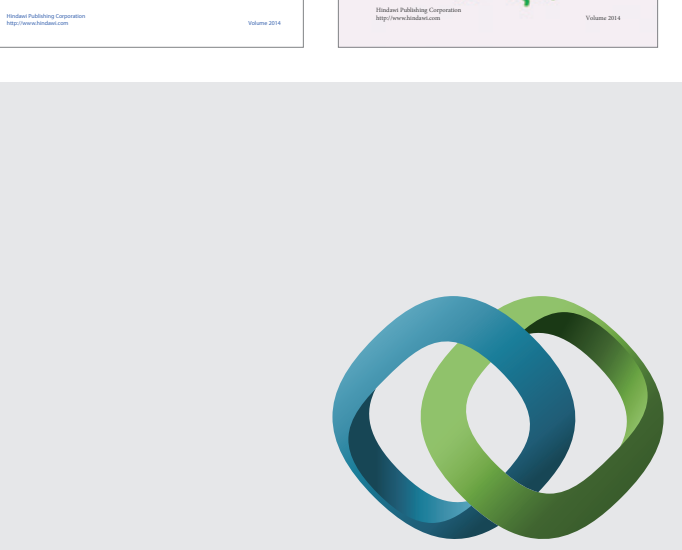

\section{Hindawi}

Submit your manuscripts at

http://www.hindawi.com
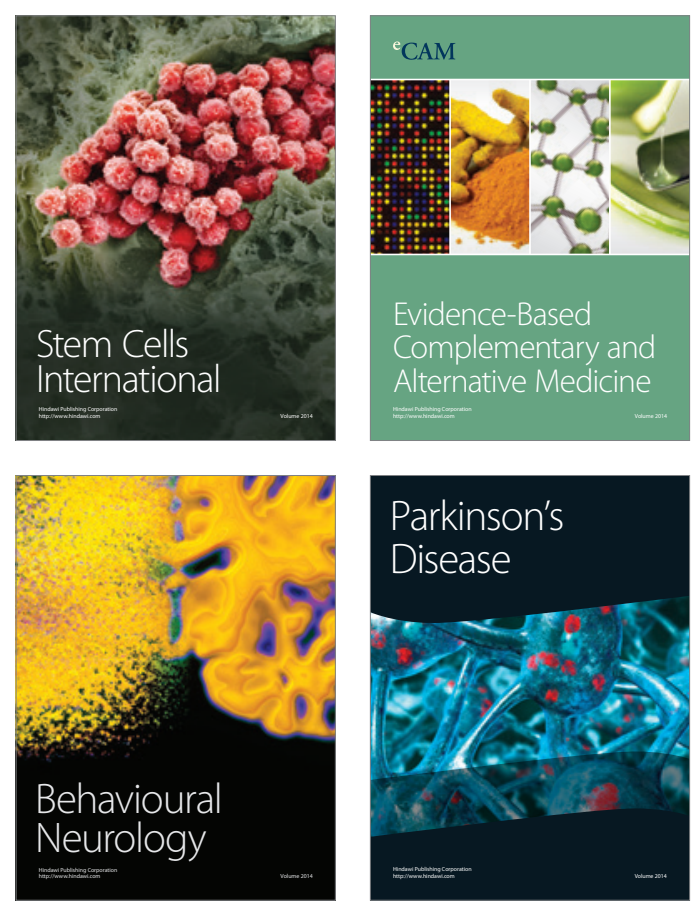

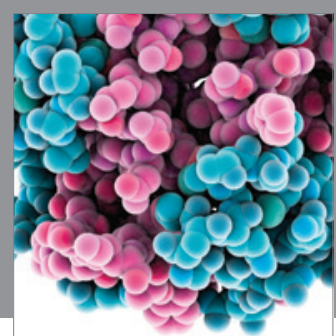

Journal of
Diabetes Research

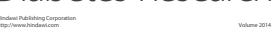

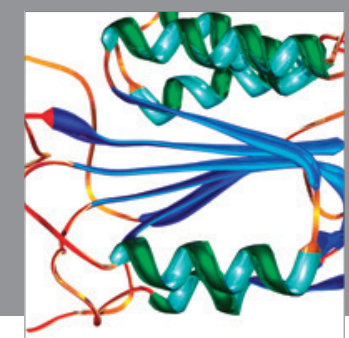

Disease Markers
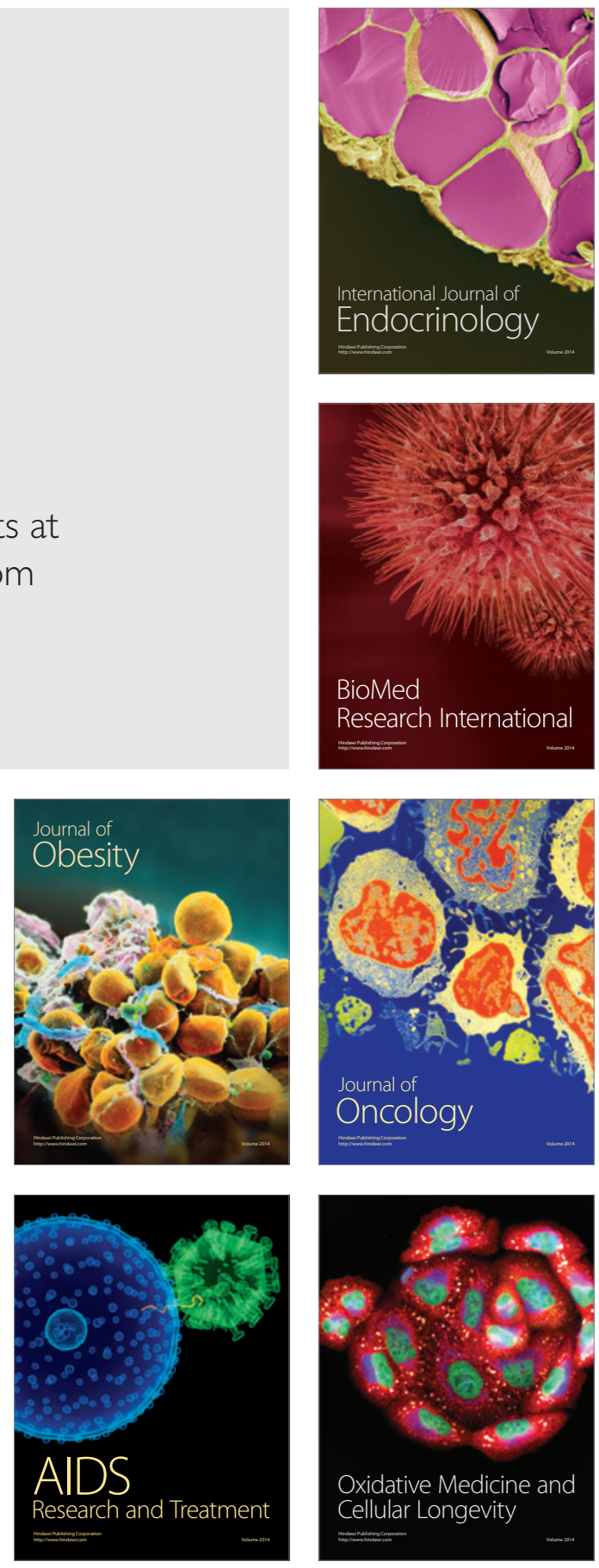